\title{
Analysis of a model for ethanol production through continuous fermentation
}

\author{
S. D. Watt ${ }^{1}$ H. S. Sidhu ${ }^{2} \quad$ M. I. Nelson ${ }^{3}$ \\ A. K. Ray $^{4}$
}

(Received 26 July 2007; revised 9 October 2007)

\begin{abstract}
We investigate an experimentally verified model for the production of ethanol through continuous fermentation. Previous studies investigated this model using direct integration. Such integration is time consuming as parameter regions of interest can only be determined through laborious and repetitive calculations. Using techniques from nonlinear dynamical systems theory, in particular a combination of steady state analysis and path following methods, practical insights into operating strategies are found. We use the performance of the reaction scheme in one tank as a benchmark for comparing the performances of multiple tanks.
\end{abstract}

See http://anziamj.austms.org.au/ojs/index.php/ANZIAMJ/article/view/322 for this article, (C) Austral. Mathematical Soc. 2007. Published October 15, 2007. ISSN 1446-8735 


\section{Contents}

1 Introduction

C86

2 Governing equations

C87

3 Results

C89

3.1 Washout conditions . . . . . . . . . . . . C89

3.2 Numerical results . . . . . . . . . . . . . . . . . . C90

4 Conclusions

C95

A Nomenclature

C96

References

C97

\section{Introduction}

The search for alternative fuels which are both economically and environmentally viable is of great current interest. One of the proposed fuel alternatives is ethanol, which can be produced from many sources including wood chips, corn husks and other agricultural waste products. Using ethanol instead of gasoline will reduce carbon emissions by more than $80 \%$ and will reduce overall gasoline consumption by $30 \%$ [7]. We investigate a model for the production of ethanol through continuous fermentation. Ethanol productivity can be increased up to four fold when ethanol is produced using continuous fermentation, compared to other methods such as centrifuges and settling tanks $[2,3]$.

We analyse a model for ethanol production that was proposed by Jarzebski [6], which is an extension of the model by Ghommidh et al. [1]. The model contains five variables representing the various cell, ethanol and sub- 
strate concentrations. The biochemical parameters were estimated from experimental results [8]. The model was analysed using a selection of different flow rates and substrate concentration of the feed using direct numerical integration. This approach is time consuming as parameter regions of interest can only be determined through laborious and repetitive calculations. More importantly, crucial regions of parameter space (in terms of optimal performance) can easily be missed by using this approach. Using techniques from nonlinear dynamical systems theory, in particular a combination of steady state analysis and path following methods, practical insights into operating strategies are found.

Our analysis shows that, although the system can exhibit interesting behaviour such as oscillatory states and period doubling, the performance of the system is nevertheless optimised by operating in a steady state regime.

\section{Governing equations}

The production of ethanol is modelled using the scheme by Jarzebski [6]. This model assumes that the tank is well mixed and there is no recycle. The cell populations are broken into three groups: viable cells $\left(X_{v}\right)$, non-viable cells $\left(X_{n v}\right)$ and dead cells $\left(X_{d}\right)$. Non-viable cells are non-growing, but still retain the ability to produce ethanol. The biological reactions are

$$
\begin{aligned}
S & \rightarrow P \\
X_{v} & \rightarrow 2 X_{v}, \\
X_{v} & \rightarrow X_{n v}, \\
X_{v} & \rightarrow X_{d}, \\
X_{n v} & \rightarrow X_{d},
\end{aligned}
$$

where $S$ and $P$ represent the substrate and ethanol respectively. The second reaction denotes viable cell division. In a single reactor, ethanol production 
is modelled using five ODEs:

$$
\begin{aligned}
V \frac{d X_{v}}{d t} & =-F X_{v}+V\left(\mu_{v}-\mu_{n v}-\mu_{d}\right) X_{v} \\
V \frac{d X_{n v}}{d t} & =-F X_{n v}+V\left(\mu_{n v} X_{v}-\mu_{d} X_{n v}\right), \\
V \frac{d X_{d}}{d t} & =-F X_{d}+V \mu_{d}\left(X_{v}+X_{n v}\right) \\
V \frac{d P}{d t} & =-F P+V\left(\frac{\mu_{v} X_{v}}{Y_{x \mid p}}+m_{p} X_{n v}\right), \\
V \frac{d S}{d t} & =F\left(S_{0}-S\right)-V\left(\frac{\mu_{v} X_{v}}{Y_{x \mid s}}+m_{s} X_{n v}\right),
\end{aligned}
$$

where $F$ is the flow rate into the tank, $V$ is the volume of the tank, $m$ is the maintenance factor, $Y$ is the yield coefficient and $S_{0}$ is the substrate concentration in the feed. Note that the maintenance terms, involving $m_{p}$ and $m_{s}$ in (4) and (5), do not appear in (2) as this process does not consume or produce non-viable cells, but produces ethanol and consumes substrate respectively. All other parameters are defined in the nomenclature in Appendix A. We assume that there is only substrate in the feed. The growth rates, which account for product inhibition and substrate limitation, are given by Jarzebski [6], which are variations of the growth rates given by Ghommidh et al. [1]:

$$
\begin{aligned}
\mu_{v} & =\mu_{\max } \frac{S}{K_{1}+S}\left(1-\frac{P}{P_{c}} \frac{S}{K_{2}+S}\right) \\
\mu_{d} & =-\mu_{\max } \frac{S}{K_{1}+S}\left(1-\frac{P}{P_{c}} \frac{S}{K_{2}+S}\right) \\
\mu_{n v} & =\mu_{\max }^{\prime} \frac{S}{K_{1}+S}\left(1-\frac{P}{P_{c}^{\prime}} \frac{S}{K_{2}+S}\right)-\mu_{v} .
\end{aligned}
$$

All reaction rates are assumed to be non-negative. If the concentration of any chemical species is such that a reaction rate would be negative, that rate is then reset to zero [1]. 


\section{Results}

Numerical results were obtained using the path following software AUTO [4]. In the corresponding figures, the standard notation is used: solid and dashed lines represent stable and unstable steady states respectively, squares are Hopf points (that is, the points where oscillatory solution branches emanate from the steady state solution), and open and solid circles represent unstable and stable periodic solutions respectively. For periodic solutions, the norm chosen is the integral over the period of the solution.

We investigate behaviour of the system as a function of the residence time, $\tau=V / F$, and substrate concentration in the feed, $S_{0}$. Note that the dilution rate $D=F / V$ was used by Jarzebski [6], the reciprocal of the residence time. The case $D=0.089 \mathrm{~h}^{-1}(\tau=11.24 \mathrm{~h})$ and $S_{0}=138 \mathrm{gl}^{-1}$ was used to estimate the biochemical parameters from the experimental data. For a dilution rate of $D=0.05 \mathrm{~h}^{-1}(\tau=20 \mathrm{~h})$ the system was shown to exhibit both steady state and oscillatory behaviour for feed substrate concentrations of $138 \mathrm{gl}^{-1}$ and $160 \mathrm{gl}^{-1}$, whereas the system only possessed steady state solutions for $100 \mathrm{gl}^{-1}$.

\subsection{Washout conditions}

In any biochemical system the washout solution must be avoided. Washout corresponds to a steady state where concentration of substrate in the influent is equal to that in the effluent. The washout steady state solution to (1)-(5) is

$$
X_{v}=X_{n v}=X_{d}=P=0 \text { and } S=S_{0} .
$$

The stability of the washout steady state solution is determined by the eigenvalues of the corresponding Jacobian matrix. Before proceeding, we first 
examine the reaction rates. In the washout state we have

$$
\mu_{v}=\frac{\mu_{\max } S_{0}}{K_{1}+S_{0}}, \quad \mu_{d}=-\frac{\mu_{\max } S_{0}}{K_{1}+S_{0}}, \quad \mu_{n v}=\frac{S_{0}}{K_{1}+S_{0}}\left(\mu_{\max }^{\prime}-\mu_{\max }\right) .
$$

Thus the value for $\mu_{d}$ has to be set to zero. The value of $\mu_{n v}$ depends on $\mu_{\max }^{\prime}$ and $\mu_{\max }$. From experimental values [8], we have $\mu_{\max }>\mu_{\max }^{\prime}$ and so $\mu_{n v}$ is also set to zero. The eigenvalues of the Jacobian matrix are $\lambda_{i}=-1 / \tau<0$, $i=1,2,3,4$, and $\lambda_{5}=\mu_{\max } \mathcal{F}-1 / \tau$ where $\mathcal{F}=S_{0} /\left(K_{1}+S_{0}\right)$. The washout state is therefore stable when

$$
\tau<\frac{1}{\mu_{\max } \mathcal{F}}=\frac{1}{\mu_{\max }}\left(1+\frac{K_{1}}{S_{0}}\right) .
$$

This corresponds to $4.12 \mathrm{~h}$ for a feed substrate concentration of $100 \mathrm{gl}^{-1}$ and $4.075 \mathrm{~h}$ for a feed substrate concentration of $160 \mathrm{gl}^{-1}$. It can be shown that when $\mu_{\max } \leq \mu_{\max }^{\prime}$, the washout solution is stable if

$$
\tau<\frac{1}{\left(2 \mu_{\max }-\mu_{\max }^{\prime}\right) \mathcal{F}} .
$$

\subsection{Numerical results}

We first consider the case when the feed substrate concentration is $100 \mathrm{gl}^{-1}$. Figure 1 shows there are no periodic solutions, but there are regions of static bistability, corresponding to low and high viable cell concentrations $\left(X_{v}\right)$. Starting from a zero residence time, the system is in the washout state until a residence time of $4.12 \mathrm{~h}$ is reached. For $\tau>4.12$, viable cells and ethanol are produced and the substrate concentration begins to diminish. When the residence time is increased to $6 \mathrm{~h}$, non-viable cells start to be produced, which decreases the number of viable cells. There is also another region of bistability for large residence times, where the viable cells are in abundance. This figure demonstrates the advantage of using path following methods as it would be difficult to find all these interesting dynamics if only 


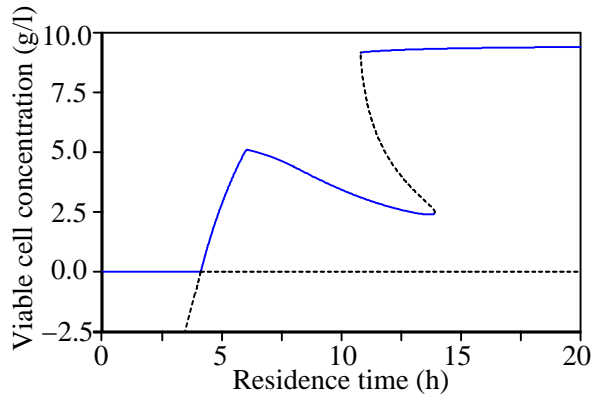

(a)

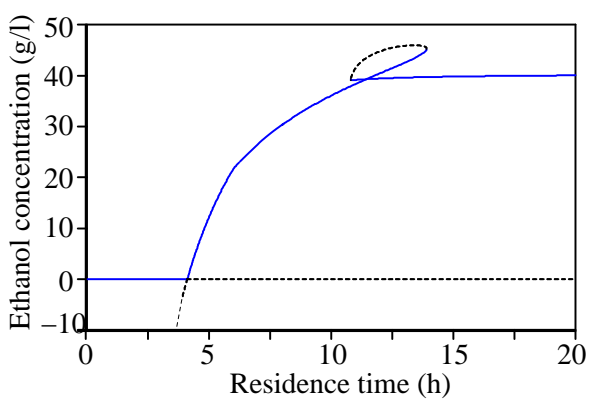

(c)

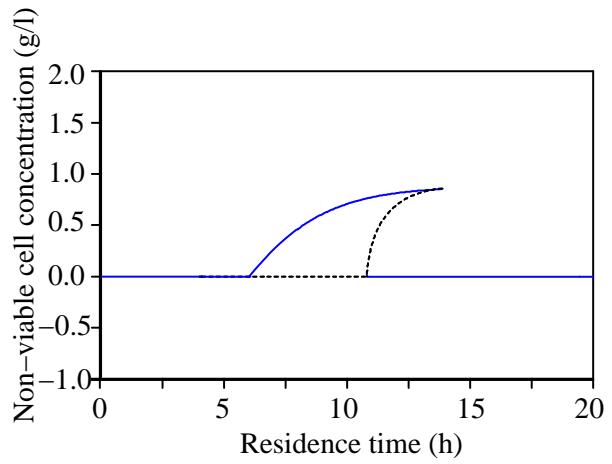

(b)

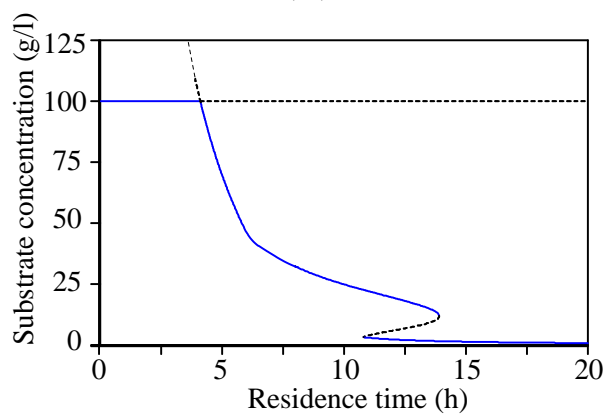

(d)

Figure 1: Steady state diagrams for (a) $X_{v}$ (b) $X_{n v}$ (c) $P$ and (d) $S$ as a function of $\tau$ when $S_{0}=100 \mathrm{gl}^{-1}$.

direct numerical integration is utilised. The maximum ethanol concentration is $P=45.25 \mathrm{gl}^{-1}$, when the residence time is $\tau=13.93 \mathrm{~h}$ (see Figure $1 \mathrm{c}$ ). The performance of chemical reactors is often characterised by the reactor productivity at the outlet,

$$
\operatorname{Pr}=\frac{P}{\tau} .
$$

Figure 2 shows the productivity as a function of the residence time. The optimal productivity is $\operatorname{Pr}=3.8 \mathrm{gl}^{-1} \mathrm{~h}^{-1}$ which occurs at a residence time $\tau=7.47 \mathrm{~h}$. Although the maximum ethanol concentration is $P=45.25 \mathrm{gl}^{-1}$, 


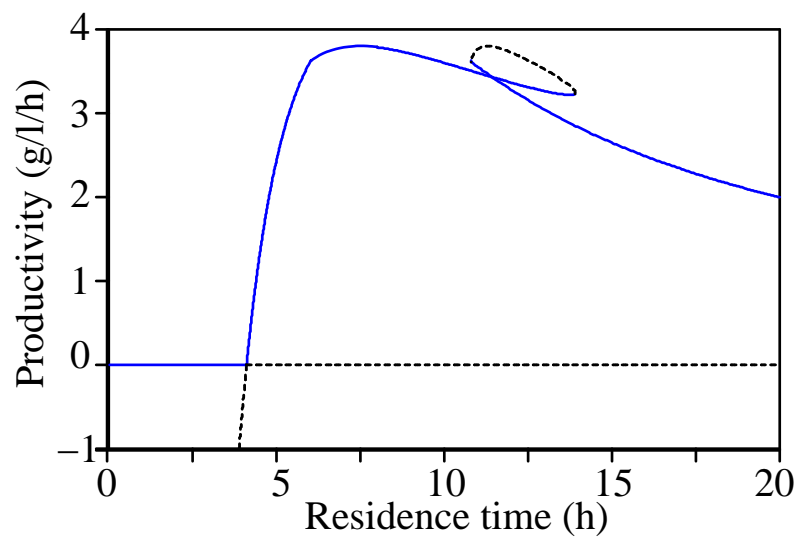

Figure 2: Productivity, $P / \tau$, versus residence time for $S_{0}=100 \mathrm{gl}^{-1}$.

when $\tau=13.93 \mathrm{~h}$, the productivity obtained at this operational condition is only $3.25 \mathrm{gl}^{-1} \mathrm{~h}^{-1}$, about $15 \%$ less than the maximum value.

Jarzebski [6] stated that periodic solutions can occur at increased feed substrate concentrations. As mentioned earlier, periodic solutions emanate from the Hopf point, and therefore it is useful to determine the Hopf locus. The location of Hopf points is shown in Figure 3 as a function of the feed substrate concentration and residence time. From Figure 3 the minimum point on the Hopf locus curve corresponds to a $H 2_{1}$ degenerate point [5] (that is, there are no Hopf points for feed substrate concentrations below this value). This occurs at a feed substrate concentration of $S_{0}=108 \mathrm{gl}^{-1}$. The graph asymptotes to a feed substrate concentration of $122 \mathrm{gl}^{-1}$ at an infinite residence time. Thus for feed substrate concentrations greater than $122 \mathrm{gl}^{-1}$ the steady state diagram contains one Hopf point, for $108 \mathrm{gl}^{-1} \leq S_{0} \leq 122 \mathrm{gl}^{-1}$ there are two Hopf points and for $S_{0}<108 \mathrm{gl}^{-1}$, there are no Hopf points on the steady state diagram.

We now consider a feed substrate concentration of $138 \mathrm{gl}^{-1}$. At this value of feed substrate concentration the system exhibits both steady state and periodic behaviour [6]. The steady state diagrams are shown in Figure 4, for 


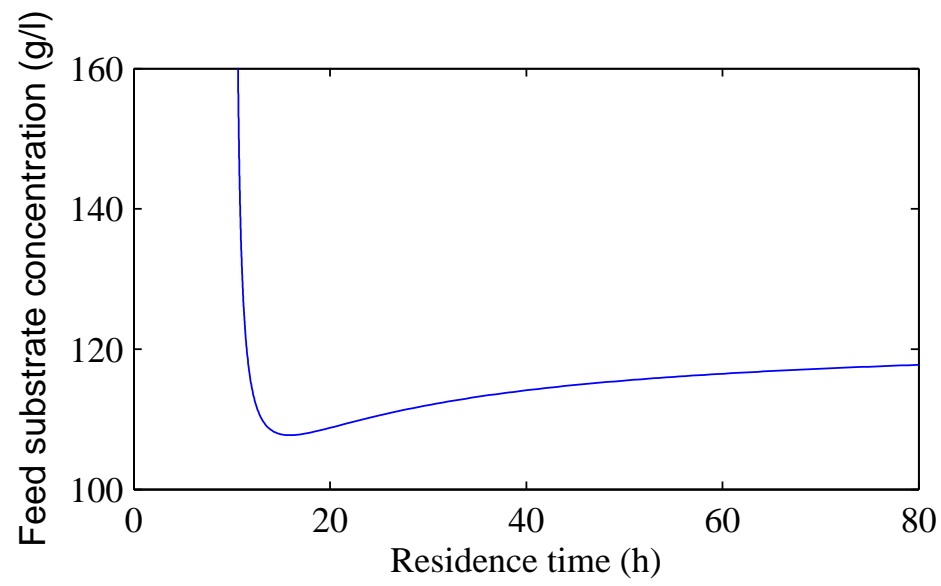

Figure 3: The Hopf locus in the $\left(\tau, S_{0}\right)$-parameter plane.

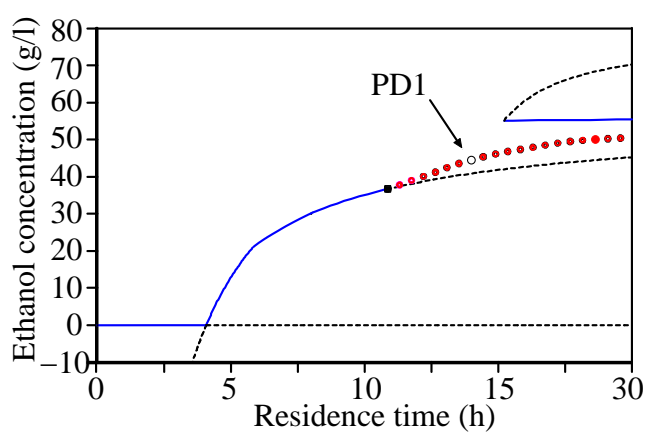

(a)

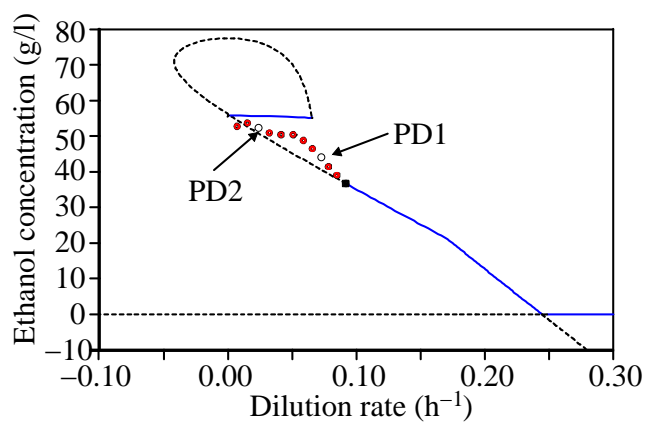

(b)

FigurE 4: The ethanol concentration as a function of (a) residence time and (b) dilution rate for a feed substrate of concentration of $138 \mathrm{gl}^{-1}$, where PD1 and PD2 denotes period doubling bifurcations. 


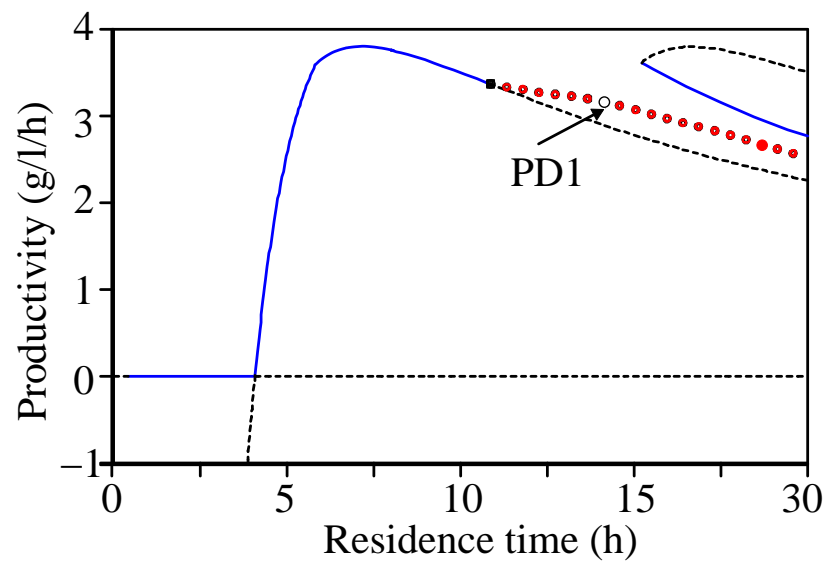

Figure 5: Productivity versus residence time for a feed substrate concentration of $138 \mathrm{gl}^{-1}$, where PD1 denotes a period doubling bifurcation.

the concentration of ethanol, and Figure 5 for the ethanol productivity. The optimal productivity is given by $\operatorname{Pr}=3.8 \mathrm{gl}^{-1}$ which occurs at a residence time of $\tau=7.167 \mathrm{~h}$. The periodic solutions become unstable at a residence time of about $13.9 \mathrm{~h}$ at a period doubling bifurcation, denoted by PD1 in Figures 4 and 5. The period doubled solution branch loses stability at a residence time of $\tau=14.27 \mathrm{~h}$ after which the system is returned to the original periodic solution branch. There is also a period doubling bifurcation at large residence times of about $55 \mathrm{~h}$, corresponding to a dilution rate $D(=$ $1 / \tau)=0.018 \mathrm{~h}^{-1}$ and is denoted by PD2 in Figure $4 \mathrm{~b}$, which exhibits a period doubling cascade route to chaos. However, since the performance in this region is sub-optimal and, for brevity, is not discussed here.

The high viable cell solution branch in Figures 4a and 5 appear 'disjointed' from the rest of the solution branch. When the dilution rate is used as the primary bifurcation parameter instead of the residence time, these high and low viable cell solutions are shown to be part of the same solution branch, as shown in Figure 4b noting that PD1 in Figures 4a and 4b correspond to the same period doubling bifurcation point and PD2 corresponds to the pe- 
riod doubling bifurcation to chaos for high residence times discussed above. Furthermore we have continued this branch into non-physical values of $D$ to show the entire solution branch. Hence, when the residence time formulation is used, changing the dilution rate from a small positive $D$ value to a small negative dilution rate corresponds to the residence time changing discontinuously from a large positive $\tau$ value to a large negative residence time.

Upon increasing the feed substrate concentration to $160 \mathrm{gl}^{-1}$ there appears a combination of stable periodic solutions and stable steady states. The optimal productivity of $3.8 \mathrm{gl}^{-1} \mathrm{~h}^{-1}$ occurs at $\tau=7.11 \mathrm{~h}$ when the system operates at a steady state condition, which was the case for feed substrate concentrations of $100 \mathrm{gl}^{-1}$ and $138 \mathrm{gl}^{-1}$.

\section{Conclusions}

We examined a model for ethanol production through continuous fermentation in a single well mixed reactor. The chemical system was based on an experimentally verified model described by Jarzebski [6], but has never been systematically analysed to determine its dynamical behaviour and optimal performance. We established the conditions for washout to occur. Since washout is undesirable, equation (6) defines the minimum residence time that can be used. We have shown that natural oscillations cannot occur if the feed substrate concentration, $S_{0}$, is sufficiently low, $S_{0}<108 \mathrm{gl}^{-1}$. We have also shown that there is one Hopf point on the steady state diagram for $S_{0}>122 \mathrm{gl}^{-1}$, whereas two Hopf points occur for $108 \mathrm{gl}^{-1}<S_{0}<122 \mathrm{gl}^{-1}$. Depending on the feed substrate concentration, the system exhibited steady state and periodic behaviour. Period doubling bifurcations, with a period doubling cascade route to chaos were observed. However, we found that for the range of feed substrate concentrations analysed by Jarzebski [6], the performance of the reactor based on ethanol productivity was maximised when the system operated at a static steady state. Furthermore, the optimal pro- 
ductivity was practically independent of the feed substrate concentration. The analysis undertaken here will be used as a benchmark for further work, when we compare the performance between the single reactor and multireactor cascade for ethanol production.

Acknowledgment This work was supported by a grant from the Australian Research Council (DP0559177).

\section{A Nomenclature}

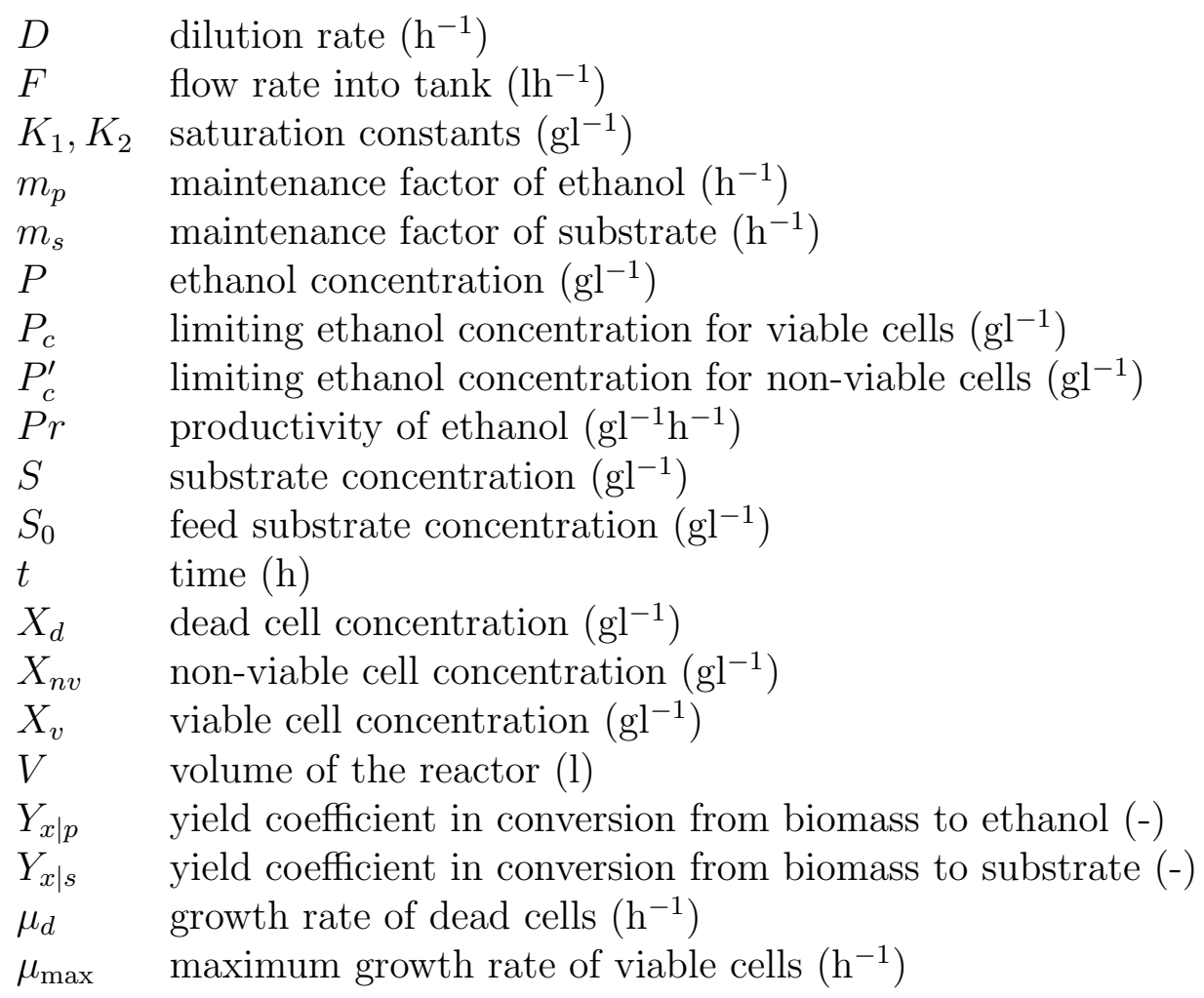


$\mu_{\max }^{\prime} \quad$ maximum growth rate on non-viable cells $\left(\mathrm{h}^{-1}\right)$

$\mu_{n v} \quad$ growth rate of non-viable cells $\left(\mathrm{h}^{-1}\right)$

$\mu_{v} \quad$ growth rate of viable cells $\left(\mathrm{h}^{-1}\right)$

$\tau \quad$ residence times $(\mathrm{h})$

Unless otherwise stated, the parameter values we use in this study are based on those given by Jarzebski [6]: $\mu_{\max }=0.25 \mathrm{~h}^{-1}, \mu_{\max }^{\prime}=0.21 \mathrm{~h}^{-1}, P_{c}=$ $70 \mathrm{gl}^{-1}, P_{c}^{\prime}=130 \mathrm{gl}^{-1}, m_{p}=2.6 \mathrm{~h}^{-1}, m_{s}=4.42 \mathrm{~h}^{-1}, Y_{x \mid p}=0.235, Y_{x \mid s}=$ 0.095 and $K_{1}=K_{2}=3 \mathrm{gl}^{-1}$.

\section{References}

[1] C. Ghommidh, J. Vaija, S. Bolarinwa and J. M. Navarro, Oscillatory behaviour of zymomonas in continuous cultures: a simple stochastic model, Biotechnology Letters 2 (9) (1989) 659-664. doi:10.1007/BF01025278 C86, C88

[2] G. R. Cysewski and C. R. Wilke, Utilization of cellulosic materials through enzymatic-hydrolysis: 1 . Fermentation of hydrolysate to ethanol and single-cell protein, Biotechnology and Bioengineering 18 (9) (1976) 1297-1313. C86

[3] G. R. Cysewski and C. R. Wilke, Rapid ethanol fermentations using vacuum and cell recycle, Biotechnology and Bioengineering 19 (8) (1977) 1125-1143. C86

[4] E. J. Doedel, T. F. Fairgrieve, B. Sandstede, A. R. Champneys, Y. A. Kuznetsov and X. Wang, AUTO97: Continuation and bifurcation software for ordinary differential equations (with hom-cont) (1998) ftp://ftp.cs.concordia.ca/pub/doedel/auto . C89 
[5] B. F. Gray and M. J. Roberts, A method for the complete qualitative analysis of two coupled ordinary differential equations on three parameters, Proceedings of the Royal Society A 416 (1988) 361-389. C92

[6] A. B. Jarzebski, Modelling of oscillatory behaviour in continuous ethanol fermentation, Biotechnology Letters 14 (2) (1992) 137-142. doi:10.1007/BF01026241 C86, C87, C88, C89, C92, C95, C97

[7] A. Lashinsky and N. D. Schwartz, How to Beat the High Cost of Gasoline. Forever!, Fortune, 153 (2) February 6 (2006) 40-47. C86

[8] L. Perego, J. M. C. D. Dias, L. H. Koshimizu, M. R. D. Cruz, W. Borzani and M. L. R. Vairo, Influence of temperature, dilution rate and sugar concentration on the establishment of steady-state in continuous ethanol fermentation of molasses, Biomass 6 (3) (1985) 247-256. C87, C90 


\section{Author addresses}

1. S. D. Watt, School of Physical, Environmental and Mathematical Sciences, University of New South Wales at the Australian Defence Force Academy, Canberra ACT 2600, Australia.

mailto:simon_watt@bigfoot.com

2. H. S. Sidhu, School of Physical, Environmental and Mathematical Sciences, University of New South Wales at the Australian Defence Force Academy, Canberra ACT 2600, Australia.

3. M. I. Nelson, School of Mathematics and Applied Statistics, University of Wollongong, NSW 2511, Australia.

4. A. K. Ray, Department of Chemical and Biochemical Engineering, University of Western Ontario, CANADA. 